\title{
Supply Chain Design in Perfect Competition
}

\author{
Terry L. Friesz and Changhyun Kwon
}

October 5, 2007

\begin{abstract}
In this paper, we apply the theory of optimal control and theory of traffic assignment for supply chain design in perfect competition. We model the time staging and generalized routing of input factors needed for production by a firm. The production process will typically involve several stages, and as such is described by paths through a production network whose nodes are the various stages of production. We develop an algorithm and test it for a small numerical example.
\end{abstract}

\section{Introduction}

In the last two decades, productions as well as consumptions has become global, firms began to recognize the importance of supply chain optimization. In sequence, supply chain management and design has become a very important issue both in practice and in research. Among many other methodologies to give optimal policy to manage supply chain, mathematical modeling and analysis of supply chain is of particular interest in scientific communities: see Beamon (1998), Min and Zhou (2002) and references therein.

In this paper, we apply the theory of optimal control (see Bryson and Ho, 1975) and theory of traffic assignment (see Friesz and Mookherjee, 2006) to a supply chain design problem. For the applications of optimal control theory in management problems, see Bensoussan et al. (1974) and Sethi and Thompson (2000) for the general introduction and see Ortega and Lin (2004) for an extensive survey. We model the time staging and generalized routing of input factors needed for production by a firm. To do so, we will employ a supply network which is connected to the production process of the firm of interest at supply-intake nodes. For the supply network, origin nodes are the sources of factor supplies while destination nodes represent the location in time and space at which factors enter the production process. The production process will typically involve several stages, and as such is described by paths through a production network whose nodes are the various stages of production. Although this perspective is reminiscent of the well known critical path method, it is substantially more general. We assume that the supply-production network just described has associated demands for finished goods that compel production activity that in turn compels the formation of supply chains within the 
supply subnetwork. Of course the supply and production processes we consider are dynamic. Kachani and Perakis (2002) describe a fluid model of the dynamic pricing and inventory management for make-to-stock manufacturing systems considering the fact that a unit of a product incurs a delay before being sold and that delay is similar to the travel times incurred in a transportation network. This same perspective is employed in this paper.

The main contributions of this paper is summarized as follows:

1. It studies supply chain as a network that unifies physical transportation and pre-processing stages.

2. It provides a mathematical model that fully describes the dynamics of flows of input factors.

3. It develops a computational method that solves the model in continuoustime and tests it for a small example.

The remainder of this paper is organized as follows. In Section 2, we begin our discussion by introducing notations for supply chain. We introduce dynamics and constraints essential in supply chain design in Section 3 adopting the theory of traffic assignment, in particular, the model in Friesz and Mookherjee (2006). Section 4 discusses the specific firm of our interest in this paper and give the final formulation for optimal supply chain design. We develop an algorithm in Section 5 and test it for a small numerical example in Section 6. Finally we conclude this paper in Section 7.

\section{Notation for the Supply Network}

We will be treating the time varying flows of production factors over a network associated with the graph $G(\mathcal{N}, \mathcal{A})$ where $\mathcal{N}$ is the relevant set of nodes and $\mathcal{A}$ is the relevant set of arcs. To model such flows, we take a path $p \in \mathcal{P}^{k}$ of the supply network to be a sequence of arcs labeled as follows:

$$
p \doteq\left\{a_{1}, a_{2}, \ldots, a_{i-1}, a_{i}, a_{i+1}, \ldots, a_{m(p)}\right\}
$$

where $\mathcal{P}^{k}$ is the set of all paths associated with the supply of production factor $k \in \mathcal{K}$ and $\mathcal{K}$ is the set of all production factors.

It will also be expedient to let $\mathcal{P}_{i j}^{k}$ denote the set of paths for movement of factor $k \in \mathcal{K}$ between supply-intake pair $(i, j) \in \mathcal{W}_{s}^{k}$. The set of all supplyintake pairs pertinent to factor $k \in \mathcal{K}$ is $\mathcal{W}_{s}^{k}$. For a given path, $p \in \mathcal{P}_{i j}^{k}$, the tail node of arc $a_{1}$ is the source of factor $k \in \mathcal{K}$, while the head node of $\operatorname{arc} a_{m(p)}$ is the location to which that same factor is delivered to the producing firm.

We denote the set of supply origin nodes by $\mathcal{N}_{O}^{k}$ and the set destination (intake) nodes by $\mathcal{N}_{D}^{k}$. Each arc traversed on the way between $(i, j) \in W_{s}^{k}$ represents either physical transportation or required pre-processing of factor $k \in \mathcal{K}$ originating at node $i \in \mathcal{N}_{O}^{k}$ prior to delivery at intake node $j \in \mathcal{N}_{D}^{k}$. 
We also let $\tau_{a_{i}}^{p k}$ be the time of exit of flow from arc $a_{i} \in p$ given shipment of factor $k \in \mathcal{K}$ at time $t$ via path $p \in \mathcal{P}^{k}$. Furthermore we take

$$
\delta_{a_{i} p}=\left\{\begin{array}{lll}
1 & \text { if } & a_{i} \in p \\
0 & \text { if } & a_{i} \notin p
\end{array}\right.
$$

to be an element of the arc-path incidence matrix.

\section{Supply Network Dynamics and Constraints}

The relevant arc dynamics for factors supplied to the sole producer are

$$
\begin{aligned}
\frac{d x_{a_{i}}^{p k}(t)}{d t} & =g_{a_{i-1}}^{p k}(t)-g_{a_{i}}^{p k}(t) \quad \forall k \in \mathcal{K}, p \in \mathcal{P}^{k}, i \in[1, m(p)] \\
x_{a_{i}}^{p k}(0) & =x_{a_{i}, 0}^{p k} \quad \forall k \in \mathcal{K}, p \in \mathcal{P}^{k}, i \in[1, m(p)]
\end{aligned}
$$

where $x_{a_{i}}^{p k}$ is the volume of factor $k$ on $\operatorname{arc} a_{i}, g_{a_{i}}^{p k}$ is flow of that factor exiting $\operatorname{arc} a_{i}$ and $g_{a_{i-1}}^{p k}$ is flow the same factor entering arc $a_{i}$ of path $p \in \mathcal{P}^{k}$. Also, $g_{a_{0}}^{p k}$ is the flow exiting the origin of path $p$; by convention we call this the flow of factor $k$ on path $p$. Furthermore

$$
x_{a}(t)=\sum_{k \in \mathcal{K}} \sum_{p \in \mathcal{P}^{k}} \delta_{a p} x_{a}^{p k}(t) \quad \forall a \in \mathcal{A}
$$

is the total arc volume ${ }^{1}$

Of course total path traversal time is

$$
C_{p}^{k}(t)=\sum_{i=1}^{m(p)}\left[\tau_{a_{i}}^{p k}(t)-\tau_{a_{i}-1}^{p k}(t)\right]=\tau_{a_{m(p)}}^{p k}(t)-t \quad \forall k \in \mathcal{K}, p \in \mathcal{P}^{k}
$$

since we use the convention that

$$
\tau_{0}^{p k}(t)=t
$$

It will prove expedient to introduce the following recursive relationships that must hold in light of the above development:

$$
\begin{aligned}
& \tau_{a_{1}}^{p k}=t+D_{a_{1}}(t) \quad \forall k \in \mathcal{K}, p \in \mathcal{P}^{k} \\
& \tau_{a_{i}}^{p k}(t)=\tau_{a_{i-1}}^{p k}(t)+D_{a_{i}}\left(\tau_{a_{i-1}}^{p}\right) \quad \forall k \in \mathcal{K}, p \in \mathcal{P}^{k}, i \in[2, m(p)]
\end{aligned}
$$

where $D_{a_{i}}(t)$ for $i \in[1, m(p)]$ is the traversal time for an arbitrary supply network arc $a_{i} \in p \in \mathcal{P}^{k}$. The dependence of arc traversal time on clock time $t$

\footnotetext{
${ }^{1}$ In expression (3) we assume that all factor flows are in comensurable units, such as pounds per second. However, it is not difficult to relax this assumption and use explicit factor weights to convert factor flows to common units of measurement.
} 
reflects the fluctuations of resources needed for transportation or preprocessing factors as they migrate through the supply network. We may easily ensure that a first-in-first out queue discipline is enforced by imposing the constraint

$$
\frac{d}{d t}\left[t+D_{a_{i}}(t)\right]=1+\frac{d}{d t} D_{a_{i}}(t)>0
$$

for an arbitrary supply network arc $a_{i} \in p \in \mathcal{P}^{k}$. This constraint assures that each exit time function is strictly monotonically increasing during periods when flow is strictly positive. Furthermore, physical reality and elementary manipulations based on the chain rule lead to the following flow propagation constraints:

$g_{a_{i}}^{p k}\left(t+D_{a_{i}}\left[x_{a_{i}}(t)\right]\right)\left(1+\frac{d}{d t} D_{a_{i}}\left[x_{a_{i}}(t)\right]\right)=g_{a_{i-1}}^{p k}(t) \quad \forall k \in \mathcal{K}, p \in \mathcal{P}^{k}, i \in[0, m(p)]$

These are proper flow propagation constraints derived in a fashion that makes them completely consistent with the chosen arc dynamics and model of arc delay. These constraints clearly involve time-dependent time shifts $D_{a_{i}}^{k}(t)$ for each supply network arc $a_{i} \in p \in \mathcal{P}^{k}$.

To discourage the early/late arrival factors of production and thereby ensure realistic behavior, we employ asymmetric early/late arrival penalties

$$
\Phi_{p}^{k}\left[t+C_{p}^{k}(x, t)-\chi_{k}\right]
$$

where $\chi_{k}$ is the desired arrival time for $p \in \mathcal{P}^{k}$. Early arrival penalties may reflect inventory costs induced by early arrivals of input factors, and late arrival penalties may be incurred by missing the deadline of productions. We also define a unit delivery fee $r_{i j}^{k}(t)$ for each supplier/intake pair for each $k \in \mathcal{K}, i \in \mathcal{N}_{O}^{k}$, $j \in \mathcal{N}_{D}^{k}$ and $t \in[0, T]$. Defining that $v_{k}$ is the economic value of time for factor $k \in \mathcal{K}$, we combine the delivery cost, actual path delays and arrival penalties to obtain

$$
\Psi_{p}^{k}(t)=\sum_{\substack{i \in \mathcal{N}_{O}^{k} \\ j \in \mathcal{N}_{D}^{k}}} r_{i j}^{k}(t)+v_{k}\left\{C_{p}^{k}(t)+\Phi_{p}^{k}\left[t+C_{p}^{k}(t)-\chi_{k}\right]\right\} \quad \forall k \in \mathcal{K}, p \in \mathcal{P}^{k}
$$

which we call the effective path cost operators. If we define

$$
E_{p}^{k}(t) \equiv t+C_{p}^{k}(t)-\chi_{k}
$$

The function

$$
\Phi_{p}^{k}\left[t+C_{p}^{k}(t)-\chi_{k}\right]=\frac{\alpha_{p}^{k}}{2}\left[E_{p}^{k}(t)\right]^{2},
$$

when $\alpha_{p}^{k} \in \Re_{++}^{1}$, is an example of a symmetric arrival penalty for $p \in \mathcal{P}^{k}$. An example of an asymmetric arrival penalty is

$$
\Phi_{p}^{k}\left[t+C_{p}^{k}(t)-\chi_{k}\right]= \begin{cases}\frac{\alpha_{p}^{k}}{2}\left[E_{p}^{k}(t)\right]^{2} & \text { if } E_{p}^{k}>0 \\ \frac{\beta_{p}^{k}}{2}\left[E_{p}^{k}(t)\right]^{2} & \text { if } E_{p}^{k}<0\end{cases}
$$


where $\beta_{p}^{k} \in \Re_{++}^{1}$ and

$$
\alpha_{p}^{k}>\beta_{p}^{k}
$$

for for $p \in \mathcal{P}^{k}$.

We also note that flow conservation requires

$$
\sum_{p \in \mathcal{P}_{i j}^{k}} \int_{0}^{T} g_{a_{m(p)}}^{p k}(t) d t=Q_{i j}^{k} \quad \forall k \in \mathcal{K}, i \in \mathcal{N}_{O}^{k}, j \in \mathcal{N}_{D}^{k}
$$

where $Q^{k}$ is the producer's demand for factor $k \in \mathcal{K}$ to arrive during the time interval $[0, T]$. Note that $T$ is not the desired arrival time, but rather a time beyond which delivery is not contemplated. The desired arrival time for factor $k \in \mathcal{K}$ is, as we have stated above, $\chi_{k}<T$. Arrival before or after $\chi_{k}$ will generally occur only if feasibility or cost considerations require so, as there is a strictly positive penalty (5) for missing the targeted delivery time. We further note that the two point boundary value problem

$$
\begin{aligned}
\frac{d y_{i j}^{k}}{d t} & =\sum_{p \in \mathcal{P}_{i j}} g_{a_{m(p)}}^{p k} & & \forall k \in \mathcal{K}, i \in \mathcal{N}_{O}^{k}, j \in \mathcal{N}_{D}^{k} \\
y_{i j}^{k}(0) & =0 & & \forall k \in \mathcal{K}, i \in \mathcal{N}_{O}^{k}, j \in \mathcal{N}_{D}^{k} \\
y_{i j}^{k}(T) & =Q_{i j}^{k} & & \forall k \in \mathcal{K}, i \in \mathcal{N}_{O}^{k}, j \in \mathcal{N}_{D}^{k}
\end{aligned}
$$

is equivalent to (6).

Naturally we suppose that there are fixed upper bounds on factor exit rates and nonnegativity restrictions for the both factor volumes and factor exit rates; that is

$$
x \geq 0 \quad G \geq g \geq 0
$$

where

$$
\begin{aligned}
x & \equiv\left(x_{a_{i}}^{p k}: k \in \mathcal{K}, p \in \mathcal{P}^{k}, i \in[1, m(p)]\right) \\
g & \equiv\left(g_{a_{i}}^{p k}: k \in \mathcal{K}, p \in \mathcal{P}^{k}, i \in[0, m(p)]\right) \\
G & =\left(G_{a_{i}}^{p k}: k \in \mathcal{K}, p \in \mathcal{P}^{k}, i \in[0, m(p)]\right)
\end{aligned}
$$

We will shortly employ the related vector notation

$$
\begin{aligned}
y & =\left(y_{i j}^{k}: k \in \mathcal{K}, i \in \mathcal{N}_{O}^{k}, j \in \mathcal{N}_{D}^{k}\right) \\
Q & =\left(Q_{i j}^{k}: k \in \mathcal{K}, i \in \mathcal{N}_{O}^{k}, j \in \mathcal{N}_{D}^{k}\right)
\end{aligned}
$$

We will also use the notation

$$
x^{0}=\left(x_{a_{i}, 0}^{p k}(0): k \in \mathcal{K}, p \in \mathcal{P}^{k}, i \in[1, m(p)]\right)
$$

to refer to the vector of initial state values. We may now define 


$$
\Omega=\{g \geq 0:,(4) \text { and (9) hold }\}
$$

which is the set of control variables that represent physically meaningful factor supply flows.

As a consequence of the preceding notation and development, we may state the constrained dynamics for our supply network model as:

$$
\begin{array}{rlrl}
\frac{d x_{a_{i}}^{p k}(t)}{d t} & =g_{a_{i-1}}^{p k}(t)-g_{a_{i}}^{p k}(t) & & \forall k \in \mathcal{K}, p \in \mathcal{P}^{k}, i \in[1, m(p)] \\
\frac{d y_{i j}^{k}}{d t} & =\sum_{p \in \mathcal{P}_{i j}} g_{a_{m(p)}}^{p k} & & \forall k \in \mathcal{K}, i \in \mathcal{N}_{O}^{k}, j \in \mathcal{N}_{D}^{k} \\
g & \in \Omega & & \\
x(0) & =x^{0} & \\
y(0) & =0 & & \\
y(T) & =Q & &
\end{array}
$$

which makes clear that the link volumes $x_{a_{i}}^{p k}$ are natural state variables while the link entrance (exit) flows $g_{a_{i}}^{p k}$ are natural control variables in our formulation.

\section{The Firm of Interest}

The producing firm serviced by our supply network seeks to maximize the net present value of its profits. We will need the following additional assumptions and definitions to model the producing firm:

1. there is perfect competition in the markets for each factor $k \in \mathcal{K}$, so the producing firm of interest is an aspatial price taker with respect to the price of each factor $k \in \mathcal{K}$, namely $\pi_{k}(t)$;

2. there is also perfect competition in the markets for output product, so the producing firm of interest is an aspatial price taker with respect to the price of output product, namely $\theta(t)$;

3. there are never any shortages of input factors;

4. the total demand rate for factor $k \in \mathcal{K}$ is endogenous and denoted by $f_{k}(t)$, while

$$
f=\left(f_{k}: k \in \mathcal{K}, p \in \mathcal{P}^{k}\right)
$$

is the vector of factor demand rates;

5. the factor demand rates obey the simple flow conservation constraints

$$
f_{k}(t)=\sum_{p \in \mathcal{P}^{k}} g_{a_{m(p)}}^{p k}(t)
$$

for all $k \in \mathcal{K}$; 
6. the producing firm's production function is

$$
F(f)
$$

which determines the output rate for each vector $f$ of input factor rates;

7. the production cost of the firm is

$$
V(F)
$$

which is the cost needed to produce the output product by the amount of $F(f)$; and

8. for simplicity we assume the firm of interest holds no inventory and accepts no backorders ${ }^{2}$.

The firm's net present value of profits, using the notation introduced above, takes the form

$$
\int_{0}^{T} e^{-\rho t}\left[\theta(t) F(f)-\sum_{k \in \mathcal{K}} \pi_{k}(t) f_{k}(t)-V(F)-\sum_{k \in \mathcal{K}} \sum_{p \in \mathcal{P}^{k}} \Psi_{p}^{k} g_{a_{m(p)}}^{p}(t)\right] d t
$$

where each term represents:

$$
\begin{aligned}
& \theta(t) F(f): \text { revenue generated by sales of final products } \\
& \sum_{k \in \mathcal{K}} \pi_{k}(t) f_{k}(t): \text { costs for the purchase of input factors } \\
& V(F): \text { production costs } \\
& \sum_{k \in \mathcal{K}} \sum_{p \in \mathcal{P}^{k}} \Psi_{p}^{k} g_{a_{m(p)}^{p}}^{p}(t): \text { supply chain path costs }
\end{aligned}
$$

The firm of our interest wants to maximize (20), hence the final formulation is:

$$
\begin{aligned}
\max J(g)=\int_{0}^{T} e^{-\rho t}[\theta(t) F(f)- & \sum_{k \in \mathcal{K}} \pi_{k}(t) f_{k}(t) \\
& \left.\quad-V(F)-\sum_{k \in \mathcal{K}} \sum_{p \in \mathcal{P}^{k}} \Psi_{p}^{k} g_{a_{m(p)}}^{p}(t)\right] d t
\end{aligned}
$$

subject to

$$
\frac{d x_{a_{i}}^{p k}(t)}{d t}=g_{a_{i-1}}^{p k}(t)-g_{a_{i}}^{p k}(t) \quad \forall k \in \mathcal{K}, p \in \mathcal{P}^{k}, i \in[1, m(p)]
$$

\footnotetext{
${ }^{2}$ Although we assume no inventory and no backorders, we can easily extend it by the same dynamics in Friesz et al. (2006).
} 


$$
\begin{gathered}
\frac{d y_{i j}^{k}}{d t}=\sum_{p \in \mathcal{P}_{i j}} g_{a_{m(p)}}^{p k} \quad \forall k \in \mathcal{K}, i \in \mathcal{N}_{O}^{k}, j \in \mathcal{N}_{D}^{k} \\
g \in \Omega, x(0)=x^{0}, y(0)=0, y(T)=Q
\end{gathered}
$$

where $\rho>0$ is the discount rate. We should note that the problem (21)-(24) is an optimal control problem with time-lags in the objective function and the state dynamics. Hence, the standard analysis of optimal control problems can not be directly used. In the next section, we provide the necessary conditions for such problems.

\subsection{The Optimal Control Problem with Time-Lags}

Prior to the development of an algorithm for solving the problem (21)-(24), we need to study necessary conditions and the gradient. We consider the following abstract optimal control problem:

$$
\min \Gamma\left[x\left(t_{f}\right), t_{f}\right]+\int_{t_{0}}^{t_{f}} F\left(x, u, u_{D}, t\right) d t
$$

subject to

$$
\begin{aligned}
\frac{d x}{d t} & =f\left(x, u, u_{D}, t\right) ; x\left(t_{0}\right)=x^{0} \\
u & \in U
\end{aligned}
$$

This is a non-standard optimal control problem, and we will need its necessary conditions. In fact we will state the following result without proof (see Friesz, 2007) :

Theorem 1 (Necessary Conditions for Optimal Control with State-Dependent Time Shifts) Suppose the following conditions hold:

(i) $u \in U \subseteq\left(L^{2}\left[t_{0}, \tau\right]\right)^{m}$;

(ii) $u_{D} \in\left(L^{2}\left[t_{0}, t_{f}\right]\right)^{m}$;

(iii) the operator $x\left(u, u_{D}, t\right):\left(L^{2}\left[t_{0}, t_{f}\right]\right)^{m} \times\left(L^{2}\left[t_{0}, \tau\right]\right)^{m} \longrightarrow\left(\mathcal{H}_{\infty}{ }^{1}\left[t_{0}, t_{f}\right]\right)^{n}$ is $\left(x^{0}, U, \Gamma\right)$-regular, continuous and $G$-differentiable ${ }^{3}$ with respect to $u$ and $u_{D}$;

(iv) $D_{i}\left(x_{i}\right):\left(\mathcal{H}^{1}\left[t_{0}, t_{f}\right]\right)^{n} \longrightarrow \mathcal{H}^{1}\left[t_{0}, t_{f}\right]$ is continuously differentiable with respect to $x_{i}$ for each $i \in[1, m]$;

(v) $\Gamma[x, t]:\left(\mathcal{H}^{1}\left[t_{0}, t_{f}\right]\right)^{n} \times \Re_{+}^{1} \longrightarrow \mathcal{H}^{1}\left[t_{0}, t_{f}\right]$ is continuously differentiable with respect to $x$;

\footnotetext{
${ }^{3}$ A reader unfamiliar with the concept of Gateaux- or G- differentiability refers to Luenberger (1969), Minoux (1986) and Jahn (1996). All other differentials in this paper are G-differentials.
} 
(vi) $F\left(x, u, u_{D}, t\right):\left(\mathcal{H}^{1}\left[t_{0}, t_{f}\right]\right)^{n} \times\left(L^{2}\left[t_{0}, \tau\right]\right)^{m} \times\left(L^{2}\left[t_{0}, \tau\right]\right)^{m} \times \Re_{+}^{1} \longrightarrow L^{2}\left[t_{0}, t_{f}\right]$ is continuously differentiable with respect to $x, u$ and $u_{D}$;

(vii) $f\left(x, u, u_{D}, t\right):\left(\mathcal{H}^{1}\left[t_{0}, t_{f}\right]\right)^{n} \times\left(L^{2}\left[t_{0}, \tau\right]\right)^{m} \times\left(L^{2}\left[t_{0}, \tau\right]\right)^{m} \times \Re_{+}^{1} \longrightarrow\left(L^{2}\left[t_{0}, t_{f}\right]\right)^{n}$ is continuously differentiable with respect to $x, u$ and $u_{D}$;

(viii) $U \subseteq\left(L^{2}\left[t_{0}, \tau\right]\right)^{m}$ is convex and compact; and

(ix) $x^{0} \in \Re^{n}$,

Then any solution $u^{*} \in U$ of the optimal control problem (25) through (27) obeys the following necessary conditions:

1. the finite dimensional variational inequality principle:

$$
\begin{gathered}
\sum_{i=1}^{m} \frac{\partial H_{1}^{*}}{\partial u_{i}}\left(u_{i}-u_{i}^{*}\right) \geq 0 \quad \forall t \in\left[t_{0}, D_{i}\left(x\left(t_{0}\right)\right)\right], u \in U \\
\sum_{i=1}^{m}\left\{\frac{\partial H_{1}^{*}}{\partial u_{i}}+\left[\frac{\partial H_{1}^{*}}{\partial\left(u_{D}\right)_{i}} \frac{1}{1+\sum_{j=1}^{m} \frac{\partial D_{i}\left(x^{*}\right)}{\partial x_{j}} \frac{d x_{j}^{*}}{d t}}\right]_{s_{i}(t)}\right\}\left(u_{i}-u_{i}^{*}\right) \geq 0 \\
\forall t \in\left[D_{i}\left(x^{*}\left(t_{0}\right)\right), t_{f}+D_{i}\left(x^{*}\left(t_{f}\right)\right)\right], u \in U
\end{gathered}
$$

where $s_{i}(t)=\arg \left[s=t-D_{i}(x(s))\right] \quad \forall t \in\left[D_{i}\left(x^{*}\left(t_{0}\right)\right), t_{f}+D_{i}\left(x^{*}\left(t_{f}\right)\right)\right], i \in$ $[1, m]$ and $H_{1}^{*}=H_{1}\left(x^{*}, u^{*}, u_{D}^{*}, \lambda^{*}, t\right)=F\left(x^{*}, u^{*}, u_{D}^{*}, t\right)+\left(\lambda^{*}\right)^{T} f\left(x^{*}, u^{*}, u_{D}^{*}, t\right)$ $\forall t \in\left[t_{0}, t_{f}\right]$

2. the state dynamics

$$
\frac{d x^{*}}{d t}=f\left(x^{*}, u^{*}, u_{D}^{*}, t\right) ; x^{*}\left(t_{0}\right)=x^{0} ; \quad \text { and }
$$

3. the adjoint dynamics

$$
(-1) \frac{d \lambda^{*}}{d t}=\nabla_{x}\left(\lambda^{*}\right)^{T} f\left(x^{*}, u^{*}, u_{D}^{*}, t\right) ; \lambda^{*}\left(t_{f}\right)=\frac{\partial \Gamma\left[x^{*}\left(t_{f}\right), t_{f}\right]}{\partial x} .
$$

The following result from Friesz (2007) is also important:

Corollary 2 (Gradient of the Criterion in the Presence of Time Shifts) When the conditions in Theorem 1 hold, the gradient of the criterion (25) is defined by

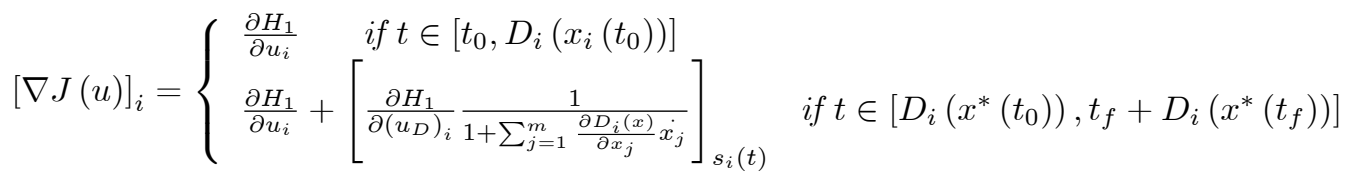

for $i=[1, m]$.

We develop a numerical method in the next section based on Theorem 1 and Corollary 2. 


\section{Descent in Hilbert Space with a Penalty Method}

We further note that the problem (21)-(24) is an instance of optimal control problems with state dependent time shifts articulated as follows:

$$
\min J=\int_{t_{0}}^{t_{f}} F\left(x, u, u_{D}, t\right) d t
$$

subject to

$$
\begin{aligned}
& x\left(u, u_{D}, t\right) \in \Omega \\
&=\left\{x: \frac{d x}{d t}=f\left(x, u, u_{D}, t\right), x(0)=0, \Gamma\left(x\left(t_{f}\right), t_{f}\right)=0, G\left(x, u, u_{D}, t\right)=0, x \geq 0\right\} \\
& \in\left(\mathcal{H}^{1}\left[t_{0}, t_{f}\right]\right)^{n}
\end{aligned}
$$

Before we apply descent in Hilbert space method to (28), we penalize the equality constraints, the terminal conditions and the non-negativity constraints as follows:

$$
\begin{aligned}
\min J=\frac{1}{2} \sum_{i} \xi_{i}\left(\Gamma_{i}\left(x\left(t_{f}\right), t_{f}\right)\right)^{2}+\int_{t_{0}}^{t_{f}} F\left(x, u, u_{D}, t\right) d t \\
\quad+\frac{1}{2} \int_{t_{0}}^{t_{f}} \sum_{i} \rho_{i} \min \left(0, x_{i}\right)^{2} d t+\frac{1}{2} \int_{t_{0}}^{t_{f}} \sum_{i} \eta_{i}\left(G_{i}\left(x, u, u_{D}, t\right)\right)^{2} d t
\end{aligned}
$$

subject to

$$
x\left(u, u_{D}, t\right) \in \tilde{\Omega}=\left\{x: \frac{d x}{d t}=f\left(x, u, u_{D}, t\right), x(0)=x_{0}\right\} \in\left(\mathcal{H}^{1}\left[t_{0}, t_{f}\right]\right)^{n},
$$

where $\xi_{i}, \rho_{i}$ and $\eta_{i}$ are increasing sequences.

This problem can be solved using a continuous time gradient projection method or a discrete time gradient projection method supplemented by spline approximations. For the penalized problem, the algorithm can be stated as following:

- Step 0. Initialization. Pick $u^{k}(t) \in U$ and set $k=0$.

- Step 1. Finding state variables. Solve the state dynamics

$$
\begin{aligned}
\frac{d x}{d t} & =f\left(x, u^{k}, u_{D}^{k}, t\right) \\
x(0) & =x_{0}
\end{aligned}
$$

and call the solution $x^{k}(t)$. 
- Step 2. Finding adjoint variables. Solve the adjoint dynamics

$$
\begin{aligned}
-\frac{d \lambda}{d t} & =\left.\nabla_{x} H^{k}\right|_{x=x^{k}} \\
\lambda\left(t_{f}\right) & =\frac{\partial \Gamma\left(x^{k}\left(t_{f}\right), t_{f}\right)}{\partial x\left(t_{f}\right)}
\end{aligned}
$$

where $H$ is the Hamiltonian for the penalized optimal control problem based on current information:

$$
\begin{aligned}
H^{k} & =F\left(x^{k}, u^{k}, u_{D}^{k}, t\right)+\frac{1}{2} \sum_{i} \rho_{i}^{k} \min \left(0, x_{i}^{k}\right)^{2} \\
& +\frac{1}{2} \sum_{i} \eta_{i}^{k}\left(G_{i}\left(x^{k}, u^{k}, u_{D}^{k}, t\right)\right)^{2}+\lambda^{T} f\left(x^{k}, u^{k}, u_{D}^{k}, t\right)
\end{aligned}
$$

Call the solution $\lambda^{k}(t)$.

- Step 3. Finding the gradient. Determine

$$
\nabla_{u} J^{k}(t)
$$

based on Corollary 2.

- Step 4. Updating the current control. For a suitably small step size

$$
\theta_{k} \in \mathbb{R}_{++}^{1}
$$

determine

$$
u^{k}(t)=P_{U}\left[u^{k}(t)-\theta_{k} \nabla_{u} J^{k}\right]
$$

- Step 5. Stopping Test. For $\epsilon \in \mathbb{R}_{++}^{1}$, a preset tolerance, stop if

$$
\left\|u^{k+1}-u^{k}\right\|<\epsilon
$$

and declare

$$
u^{*} \approx u^{k+1}
$$

Otherwise set $k=k+1$ and go to Step1.

In the following section, we provide a numerical example solved by the above algorithm.

\section{A Numerical Example}

As an illustration of the mathematical formulation (21)-(24) for supply chain design, let us consider the firm with two input factors for an output product on a 4 nodes and 3 arcs network presented in Figure ??: 


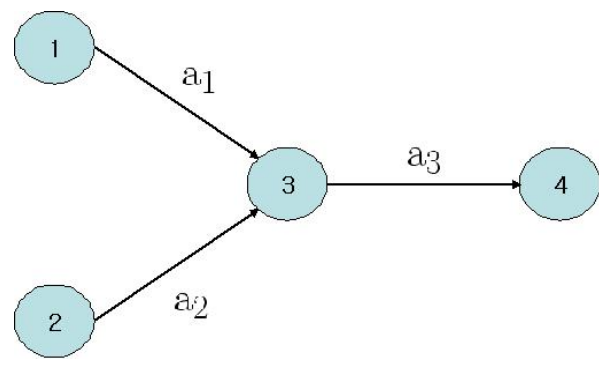

4-node, 3-arc network

Let us assume input factor $k_{1}$ is supplied through node 1, 3 and 4, and input factor $k_{2}$ is supplied through node 2,3 and 4 . That is,

$$
\begin{array}{ll}
\mathcal{P}^{k_{1}}=\left\{p_{1}\right\} ; & p_{1}=\left\{a_{1}, a_{3}, a_{4}\right\} \\
\mathcal{P}^{k_{2}}=\left\{p_{2}\right\} ; & p_{2}=\left\{a_{2}, a_{3}, a_{4}\right\}
\end{array}
$$

We consider the time horizon of $[0, T]=[0,10]$ and the firm need each factor delivered by

$$
\begin{aligned}
& \chi_{1} \stackrel{\circ}{=} \chi_{k_{1}}=7 \\
& \chi_{2} \stackrel{\circ}{=} \chi_{k_{2}}=9
\end{aligned}
$$

The firm's demand for each input factor is

$$
\begin{aligned}
& Q_{1} \stackrel{\circ}{=} Q_{1,4}^{k_{1}}=15 \\
& Q_{2} \stackrel{\circ}{=} Q_{2,4}^{k_{2}}=10
\end{aligned}
$$

The price of each factor is

$$
\begin{aligned}
\pi_{1} \stackrel{\circ}{=} \pi_{k_{1}} & =10 \exp \left(\frac{t}{20}\right) \\
\pi_{2} \stackrel{\circ}{=} \pi_{k_{2}} & =5 \exp \left(\frac{t}{10}\right)
\end{aligned}
$$

and the price of output product is

$$
\theta(t)=30 \exp \left(\frac{t}{15}\right)
$$

Let the production function

$$
\begin{gathered}
F(f)=f_{1}+2 f_{2} \\
f_{1} \stackrel{\circ}{=} f_{k_{1}} \\
f_{2} \stackrel{\circ}{=} f_{k_{2}}
\end{gathered}
$$


and the production cost function

$$
V(F)=\frac{1}{10} F^{2}=\frac{1}{10}\left(f_{1}^{2}+4 f_{1} f_{2}+4 f_{2}^{2}\right)
$$

We rewrite the arc flow at each arc at each path

$$
\begin{aligned}
& x_{1} \stackrel{\circ}{=} x_{a_{1}}=x_{a_{1}}^{p_{1}, k_{1}} \\
& x_{2} \stackrel{\circ}{=} x_{a_{2}}=x_{a_{2}}^{p_{2}, k_{2}} \\
& x_{31} \stackrel{\circ}{=} x_{a_{3}}^{p_{1}, k_{1}} \\
& x_{32} \stackrel{\circ}{=} x_{a_{3}}^{p_{2}, k_{2}} \\
& x_{3} \stackrel{\circ}{=} x_{a_{3}}=x_{31}+x_{32}
\end{aligned}
$$

and the arc delay at each arc

$$
\begin{aligned}
& D_{1} \stackrel{\circ}{=} D_{a_{1}}=1+\frac{x_{1}}{10} \\
& D_{2} \stackrel{\circ}{=} D_{a_{2}}=2+\frac{x_{2}}{15} \\
& D_{3} \stackrel{\circ}{=} D_{a_{3}}=1+\frac{x_{3}}{20}
\end{aligned}
$$

The delivery fee at each O-D pair is

$$
\begin{aligned}
& r_{1} \stackrel{\circ}{=} r_{12}=2 \\
& r_{2} \stackrel{\circ}{=} r_{23}=1 \\
& r_{3} \stackrel{\circ}{=} r_{34}=3
\end{aligned}
$$

and the time value of each factor is

$$
\begin{aligned}
& v_{1} \stackrel{\circ}{=} v_{k_{1}}=2 \\
& v_{2} \stackrel{\circ}{=} v_{k_{2}}=1
\end{aligned}
$$

Then the total transversal time is

$$
\begin{aligned}
& C_{1} \stackrel{\circ}{=} C_{p_{1}}^{k_{1}}=D_{1}+D_{3}=2+\frac{x_{1}}{10}+\frac{x_{3}}{20} \\
& C_{2} \stackrel{\circ}{=} C_{p_{2}}^{k_{2}}=D_{2}+D_{3}=3+\frac{x_{2}}{15}+\frac{x_{3}}{20}
\end{aligned}
$$

Let us define the symmetric early/late penalty function

$$
\begin{aligned}
\Phi_{1}\left[t+C_{1}-\chi_{1}\right] & \stackrel{\circ}{=} \Phi_{p_{1}}^{k_{1}}\left[t+C_{p_{1}}^{k_{1}}(t)-\chi_{k_{1}}\right] \\
& =\frac{1}{5}\left(t+2+\frac{x_{1}}{10}+\frac{x_{3}}{20}-\chi_{1}\right)^{2} \\
\Phi_{2}\left[t+C_{2}-\chi_{2}\right] & \stackrel{\circ}{=} \Phi_{p_{2}}^{k_{2}}\left[t+C_{p_{2}}^{k_{2}}(t)-\chi_{k_{2}}\right] \\
& =\frac{1}{5}\left(t+3+\frac{x_{2}}{15}+\frac{x_{3}}{20}-\chi_{2}\right)^{2}
\end{aligned}
$$


Then we have the effective delay operator

$$
\begin{aligned}
& \Psi_{1}(t) \stackrel{\circ}{=} \Psi_{k_{1}}^{p_{1}}(t)=r_{1}+r_{3}+v_{1}\left\{2+\frac{x_{1}}{10}+\frac{x_{3}}{20}\right. \\
& \left.+\frac{1}{5}\left(t+2+\frac{x_{1}}{10}+\frac{x_{3}}{20}-\chi_{1}\right)^{2}\right\} \\
& \Psi_{2}(t) \stackrel{\circ}{=} \Psi_{k_{2}}^{p_{2}}(t)=r_{2}+r_{3}+v_{2}\left\{3+\frac{x_{2}}{15}+\frac{x_{3}}{20}\right. \\
& \left.+\frac{1}{5}\left(t+3+\frac{x_{2}}{15}+\frac{x_{3}}{20}-\chi_{2}\right)^{2}\right\}
\end{aligned}
$$

For the simplicity, we re-write the each flow rate and its upper bound are

$$
\begin{aligned}
& g_{1} \stackrel{\circ}{=} g_{a_{1-1}}^{p_{1} k_{1}} \leq G_{1}=10 \\
& g_{2} \stackrel{\circ}{=} g_{a_{1}}^{p_{1} k_{1}} \leq G_{2}=10 \\
& g_{3} \stackrel{\circ}{=} g_{a_{3}}^{p_{1} k_{1}} \leq G_{3}=10 \\
& h_{1} \stackrel{\circ}{=} g_{a_{2-1}}^{p_{2} k_{2}} \leq H_{1}=10 \\
& h_{2} \stackrel{\circ}{=} g_{a_{2}}^{p_{2} k_{2}} \leq H_{2}=10 \\
& h_{3} \stackrel{\circ}{=} g_{a_{3}}^{p_{2} k_{2}} \leq H_{3}=10
\end{aligned}
$$

Then the input factor to production process is

$$
\begin{aligned}
& f_{1}=g_{3} \\
& f_{2}=h_{3}
\end{aligned}
$$

and the problem becomes

$$
\begin{aligned}
\max & J=\int_{0}^{T} e^{-\rho t}\left[\theta(t) F\left(g_{3}, h_{3}\right)-\pi_{1}(t) g_{3}(t)-\pi_{2}(t) h_{3}(t)\right. \\
& \left.-V\left(g_{3}, h_{3}\right)-\Psi_{1} g_{3}(t)-\Psi_{2} h_{3}(t)\right] d t
\end{aligned}
$$

subject to

$$
\begin{aligned}
\frac{d x_{1}}{d t} & =g_{1}-g_{2} \\
\frac{d x_{2}}{d t} & =h_{1}-h_{2} \\
\frac{d x_{3}}{d t} & =g_{2}+h_{2}-g_{3}-h_{3} \\
\frac{d y_{1}}{d t} & =g_{3} \\
\frac{d y_{2}}{d t} & =h_{3}
\end{aligned}
$$




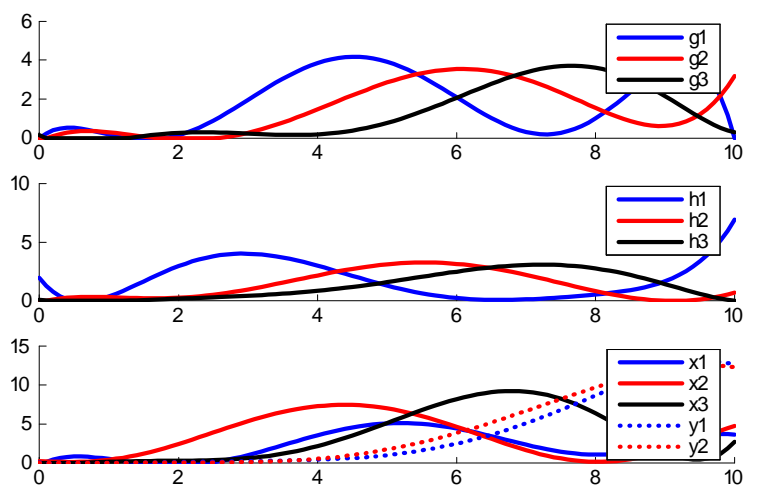

Figure 1: Optimal Supply Chain Design

$$
\begin{aligned}
g_{1}\left(t+D_{1}\left(x_{1}\right)\right)\left(1+\frac{d}{d t} D_{1}(t)\right) & =g_{2}(t) \\
g_{2}\left(t+D_{3}\left(x_{3}\right)\right)\left(1+\frac{d}{d t} D_{3}(t)\right) & =g_{3}(t) \\
h_{1}\left(t+D_{2}\left(x_{2}\right)\right)\left(1+\frac{d}{d t} D_{2}(t)\right) & =h_{2}(t) \\
h_{2}\left(t+D_{3}\left(x_{3}\right)\right)\left(1+\frac{d}{d t} D_{3}(t)\right) & =h_{3}(t) \\
0 & \leq g_{i} \leq G_{i}, 0 \leq h_{i} \leq H_{i} \quad i=1,2,3 \\
x_{1}(0) & =x_{2}(0)=x_{3}(0)=0 \\
y_{1}(0) & =y_{2}(0)=0 \\
y_{1}(T) & =Q_{1} \\
y_{2}(T) & =Q_{2}
\end{aligned}
$$

This problem is solved by MATLAB 7.0 in a generic personal computer with Intel Pentium $4 \mathrm{CPU} 2.80 \mathrm{GHz}$ and $2.00 \mathrm{~GB}$ of RAM. The result is shown in Figure 1, which shows the flow propagation of input factors over the supply chain network and accumulation of input factors.

\section{Concluding Remarks}

We have developed a mathematical formulation for optimal supply chain design considering time-lags among supply posts which may represent either physical transportation or pre-processing of input factors. The model was an instance of 
optimal control problems, in particular, with state-dependent time-lags. Based on the necessary conditions and the gradient information for such problems, we developed a numerical algorithm for solving optimal supply chain design problems, which is tested by a simple numerical example.

While this paper takes an important step to develop and analyze a continuoustime mathematical model adopting the theory of traffic assignment, further research must be conducted to resolve practical issues such as the curse of dimensionality on large-scale supply chain networks. In addition, topics of uncer-

tainty including robustness of solution and disruption management will extend this paper.

\section{References}

Beamon, B. (1998), 'Supply chain design and analysis: models and methods', International Journal of Production Economics, Vol. 55, No. 3, pp. 281-294.

Bensoussan, A., Jr., E.G.H. and Naslund, B. (1974), Management Applications of Modern Control Theory, North-Holland Publishing Company.

Bryson, A.E. and Ho, Y.C. (1975), Applied Optimal Control, Hemisphere Publishing Company.

Friesz, T.L. and Mookherjee, R. (2006), 'Solving the dynamic network user equilibrium with state-dependent time shifts', Transportation Research Part B, Vol. 40, pp. 207-229.

Friesz, T.L. (2007), Dynamic Optimization and Differential Games, Springer, forthcoming.

Friesz, T.L., Rigdon, M.A. and Mookherjee, R. (2006), 'Differential variational inequalities and shipper dynamic oligopolistic network competition', Transportation Research Part B, Vol. 40, pp. 480-503.

Jahn, J. (1996), Introduction to the Theory of Nonlinear Optimization, Springer.

Kachani, S. and Perakis, G. (2002), 'A fluid model of dynamic pricing and inventory management for make to stock manufacturing systems', Technical report, Sloan School of Management, MIT.

Luenberger, D.G. (1969), Optimization by Vector Space Methods, John Wiley and Sons, New York.

Min, H. and Zhou, G. (2002), 'Supply chain modeling: past, present and future', Computers $\mathscr{E}$ Industrial Engineering, Vol. 43, No. 1-2, pp. 231-249.

Minoux, M. (1986), Mathematical Programming: Theory and Algorithms, John Wiley and Sons. 
Ortega, M. and Lin, L. (2004), 'Control theory applications to the productioninventory problem: a review', International Journal of Production Research, Vol. 42, No. 11, pp. 2303-2322.

Sethi, S. and Thompson, G. (2000), Optimal Control Theory: Applications to Management Science and Economics, Kluwer Academic Publishers. 